\title{
Proposition d'une lecture territoriale des «flux agricoles»
}

Understanding agricultural flows through a territorial view

Überlegungen zur raümlichen Flexibilität in der Landwirtschaft

Didier Josselin, Claude Janin et Jérôme Bolot

\section{(2) OpenEdition}

Journals

Édition électronique

URL : http://journals.openedition.org/rge/4338

DOI : $10.4000 /$ rge.4338

ISSN : $2108-6478$

Éditeur

Association des géographes de l'Est

Édition imprimée

Date de publication : 1 septembre 1999

ISSN : 0035-3213

\section{Référence électronique}

Didier Josselin, Claude Janin et Jérôme Bolot, « Proposition d'une lecture territoriale des «flux agricoles» », Revue Géographique de l'Est [En ligne], vol. 39 / 4 | 1999, mis en ligne le 02 septembre 2013, consulté le 08 septembre 2020. URL : http://journals.openedition.org/rge/4338 ; DOI : https:// doi.org/10.4000/rge.4338

Ce document a été généré automatiquement le 8 septembre 2020.

Tous droits réservés 


\title{
Proposition d'une lecture territoriale des «flux agricoles»
}

\author{
Understanding agricultural flows through a territorial view \\ Überlegungen zur raümlichen Flexibilität in der Landwirtschaft
}

Didier Josselin, Claude Janin et Jérôme Bolot

1 L'aménagement du territoire rural, à travers la lutte contre la désertification des campagnes et la déprise agricole, constitue un enjeu important pour les collectivités territoriales. Cet enjeu s'accroît notamment dans les zones rurales ou de montagne, où gestion paysagère et tourisme sont des éléments essentiels du développement local (Laurent, 1994 ; Janin, 1998). C'est le cas de la Région Rhône-Alpes, où de nombreuses études ont été réalisées dans ce domaine (DRAF, 1994, Janin, 1995, Josselin, 1995, Laurent et Mundler, 1995).

2 Dans ce contexte, et compte tenu des informations disponibles dans le domaine de l'agriculture, il peut être intéressant d'étudier de plus près l'emprise de l'agriculture sur le territoire (Benoît, Deffontaines et Moisan, 1982). Le phénomène de dispersion des terres agricoles est-il important? Peut-on en dégager des formes caractéristiques? Quels en sont les déterminants principaux? Autant de questions auxquelles il semble opportun de répondre si l'on souhaite dégager des pistes pour la mise en place d'actions concrètes de gestion de l'espace (Janin, 1997, 1998). Car même si le nombre d'agriculteurs diminue, ils ont encore en charge une grande partie de l'espace rural. À ce titre, la compréhension de l'exploitation agricole en relation avec son territoire agricole demeure une clé d'analyse (Benoît, 1985).

3 Compte tenu des informations potentiellement disponibles, nous proposons ainsi deux approches complémentaires pour appréhender l'emprise agricole. Une première analyse consiste à mesurer cette emprise en considérant la commune comme entité géographique de référence, avec ce qu'elle porte d'histoire et de contraintes administratives.

4 Une seconde approche consiste à étudier le parcellaire des exploitations au sein d'entités géographiques agricoles supportant l'emprise. Ces entités se justifient par leur 
homogénéité des points de vue géomorphologique et agricole. À partir d'exemples plus précis, nous tentons d'identifier quelques formes et quelques déterminants de ces flux agricoles.

\section{Le découpage communal comme support à la mesure de l'emprise agricole}

5 Une première approche consiste à mesurer l'emprise agricole sur la base d'un découpage administratif: par exemple, celui des communes. Cette analyse peut s'appuyer sur un ensemble de sources d'information: inventaire communal, Recensement Général Agricole, fichiers de la Politique Agricole Commune, données de la Mutuelle Sociale Agricole, enquêtes locales, etc. (Cavailhès, 1992).

\section{A. Deux grands types d'information}

\section{Le RGA (Recensement Général Agricole)}

6 Parmi les données disponibles, l'une des plus utilisées demeure le RGA. Ce dernier recense, par commune, un ensemble de descripteurs relatifs aux exploitations agricoles. Une des variables, souvent utilisée pour réaliser des diagnostics territoriaux à l'échelle de la commune (par canton, département ou région, notamment) et importante lorsqu'on s'intéresse à la quantification de l'emprise agricole, est la Surface Agricole Utile. Ainsi, la SAU d'une commune A correspond à la somme des surfaces exploitées par l'ensemble des agriculteurs ayant leurs sièges d'exploitation sur cette même commune $\mathrm{A}$, et ce indépendamment de la localisation réelle des surfaces en question.

7 Cependant, le nombre d'agriculteurs diminue et les surfaces qu'ils exploitent s'accroissent en moyenne. Les jeux de reprise de terre et de concurrence foncière favorisent le morcellement. Le territoire des exploitations agricoles est de plus en plus dispersé. Ainsi, les raisons sont diverses : certaines productions nécessitent de grandes surfaces, la topographie peut contraindre fortement les pratiques. Le territoire d'une exploitation peut donc être réparti sur plusieurs communes (Josselin et Laurent, 1997). De ce fait, certaines informations du RGA (dont la SAU), très fiables si l'on s'intéresse uniquement aux exploitations comme sièges administratifs, ne sont pas adaptées à l'analyse spatiale du territoire agricole qu'elles concernent (Mergoil, 1991, Josselin, 1995). C'est le cas notamment de la SAU, mais aussi de tous les autres descripteurs agricoles qui y sont liés (nombre de têtes de bétail pour calculer des chargements, par exemple).

8 Il est donc nécessaire de recourir à d'autres types de fichiers qui prennent en compte la localisation réelle des parcelles exploitées, indépendamment de la localisation des sièges.

\section{Des données sur les « flux intercommunaux »}

9 Ces informations nous ont été fournies par l'office National Interprofessionnel des Céréales sur le département de l'Isère. Situé en région Rhône-Alpes, ce département 
associe des agricultures et des physionomies du relief variées (zones de coteaux et de plaine au nord du département et zone de montagne au sud).

Il s'agit en fait du dépouillement des dossiers de demande de paiements compensatoires dans le cadre de la Politique Agricole Commune. Deux types d'information existent, selon le type de montant compensatoire demandé. Nous ne présentons ici que celles qui concernent les exploitants agricoles dont la production céréalière déclarée est supérieure au seuil d'obligation de gel (dossiers « jaunes » de la Politique Agricole Commune) (Saillant, 1996). Elles regroupent un grand nombre de grandes exploitations ayant une forte production de céréales oléagineux et protéagineux. Les petits producteurs céréaliers ou les exploitants en polycultureélevage, souvent situés en zones de montagne, sont exclus de ce fichier. La différenciation spatiale assez forte des types de productions dans le département amène à une bipartition Nord-Sud qui oppose plaine et montagne, alors qu'en termes de flux globaux (si l'on intégrait les exploitations de montagne, par exemple), cette répartition serait probablement nettement moins marquée.

Les flux agricoles intercommunaux ont des statuts variés. Il peut être intéressant d'en donner quelques définitions.

\section{B. Flux « internes », « entrants » et « sortants »}

12 Dans notre acception, l'emprise agricole caractérise uniquement le fait qu'un agriculteur utilise une surface agricole localisée dans une commune donnée. La notion de flux contient intrinsèquement une dimension spatiale forte, à travers l'élément même qui subit le déplacement (homme, matière, information) et les objets reliés (villes, serveurs informatiques), un objet de départ du flux et un objet d'arrivée. À un second degré d'analyse, plus thématique, les flux correspondent pratiquement à des déplacements d'hommes, d'animaux ou de matériels nécessaires à l'exploitation d'une parcelle agricole. Ici, les objets géographiques (de départ et d'arrivée) sont des communes, les flux des quantités de surfaces agricoles. S'ils se réalisent à l'intérieur de la commune, ils sont internes. S'ils relient deux communes différentes, ils sont externes, plus précisément sortants pour la commune de départ et entrants pour la commune d'arrivée (fig. 1). Par commune, la somme des flux entrants et des flux internes composent la SAU réelle de la commune (dans le cas où nous disposerions de tous les flux pour le calcul). Notons que le phénomène étudié a de fortes similitudes avec les migrations alternantes (Schmitt, 1992, Caro, 1997). 
Fig. 1 : Exemple des flux agricoles entrants et sortants dans une commune française (céréales, oléagineux et protéagineux)

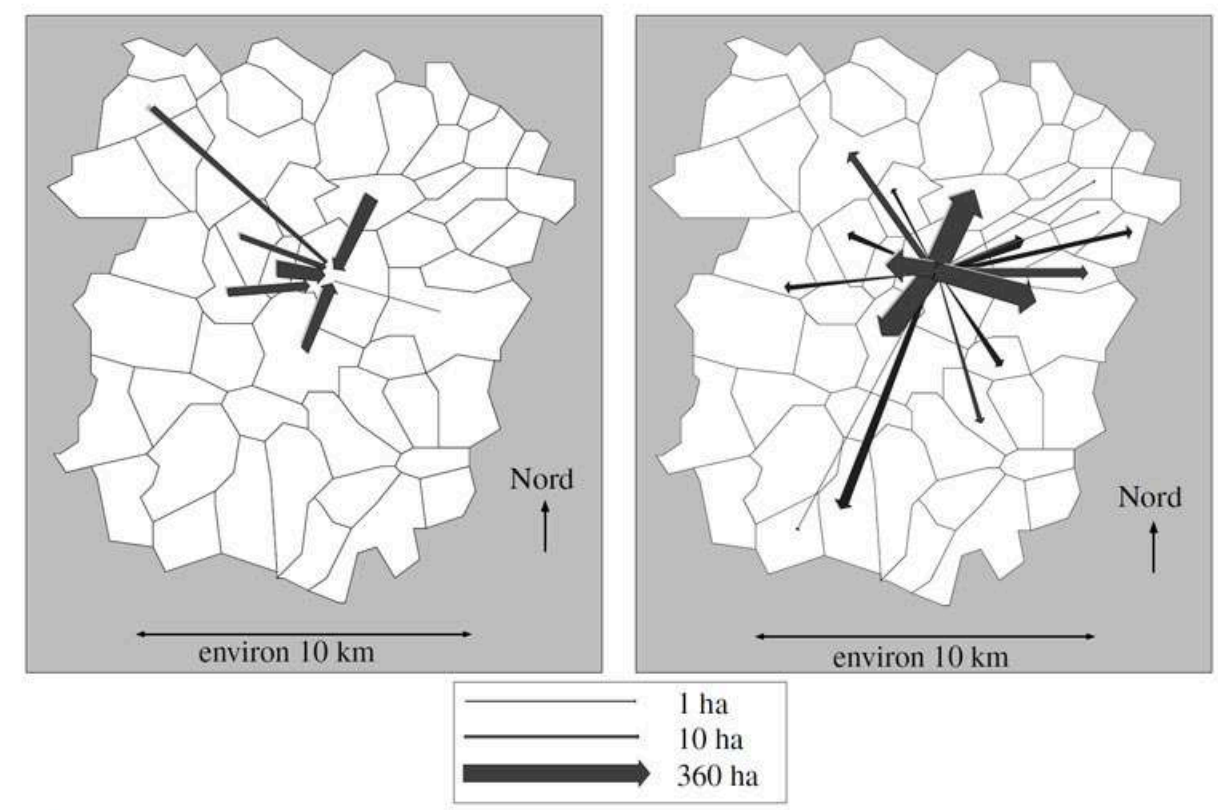

D. Josselin. J. Maillardet, THEMA-CNRS Besançon

Données PAC

13 Sur la base du découpage communal, nous définissons donc un flux agricole intercommunal entre $A$ et $B$ comme une quantité de surface agricole exploitée dans une commune $B$ par un ensemble d'agriculteurs ayant leurs sièges administratifs dans une commune $A$.

La quantification des flux - qui nous le rappelons, ne concerne qu'une partie des exploitations agricoles - montre l'importance des flux externes (entrants et sortants), dont il est nécessaire de tenir compte pour l'étude de l'emprise agricole (fig. 3, 4 et 5). En effet, si la connaissance de la SAU réelle exploitée sur chaque commune est un élément important, la connaissance des flux agricoles, même à travers le filtre du découpage administratif, l'est tout autant. 
Fig. 2 : Flux agricoles internes aux communes de l'Isère

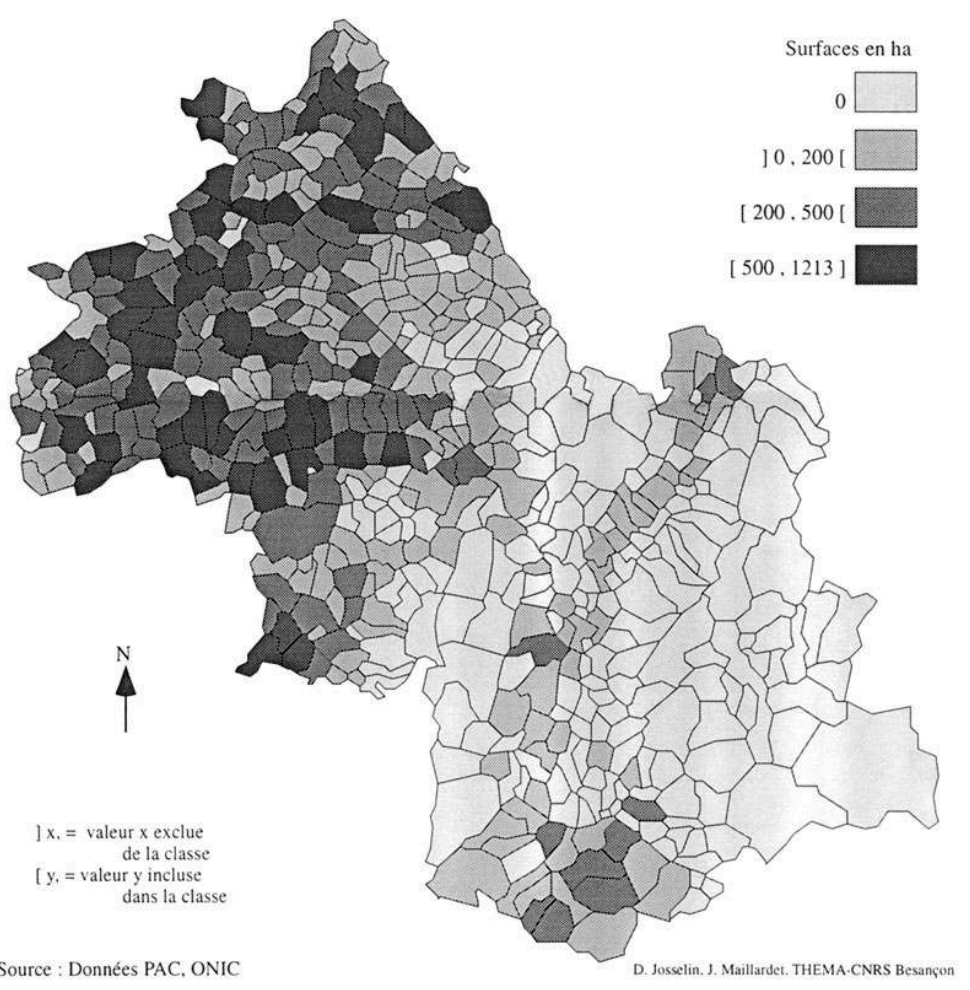

Données PAC, dossiers « jaunes ».

"Les agriculteurs ayant leur siège administratif sur une commune donnée y exploitent $n$ hectares de terres». 
Fig. 3 : Flux agricoles sortants des communes de l'Isère

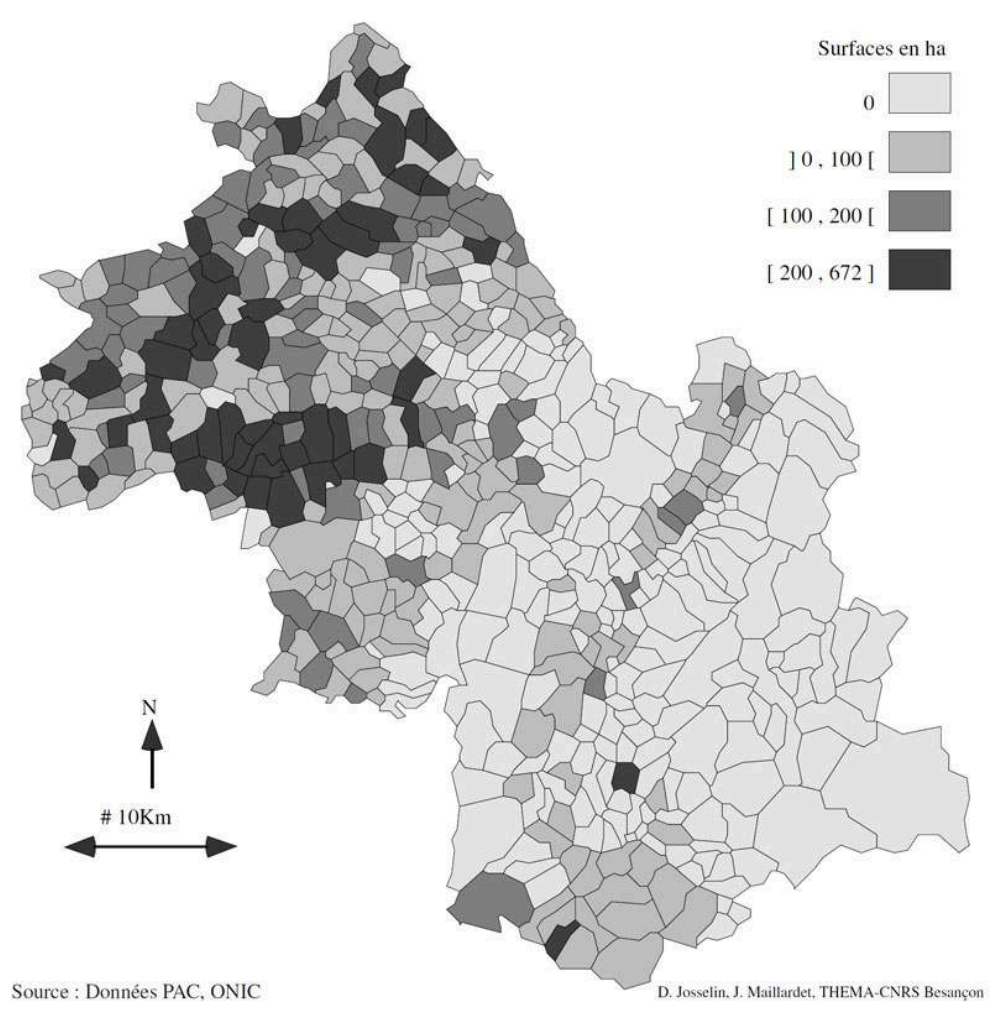

Données PAC, dossiers « jaunes ».

"Les agriculteurs ayant leur siège administratif sur une commune donnée exploitent $\mathrm{n}$ hectares de terres » dans d'autres communes ». 
Fig. 4 : Flux agricoles entrants dans les communes de l'Isère

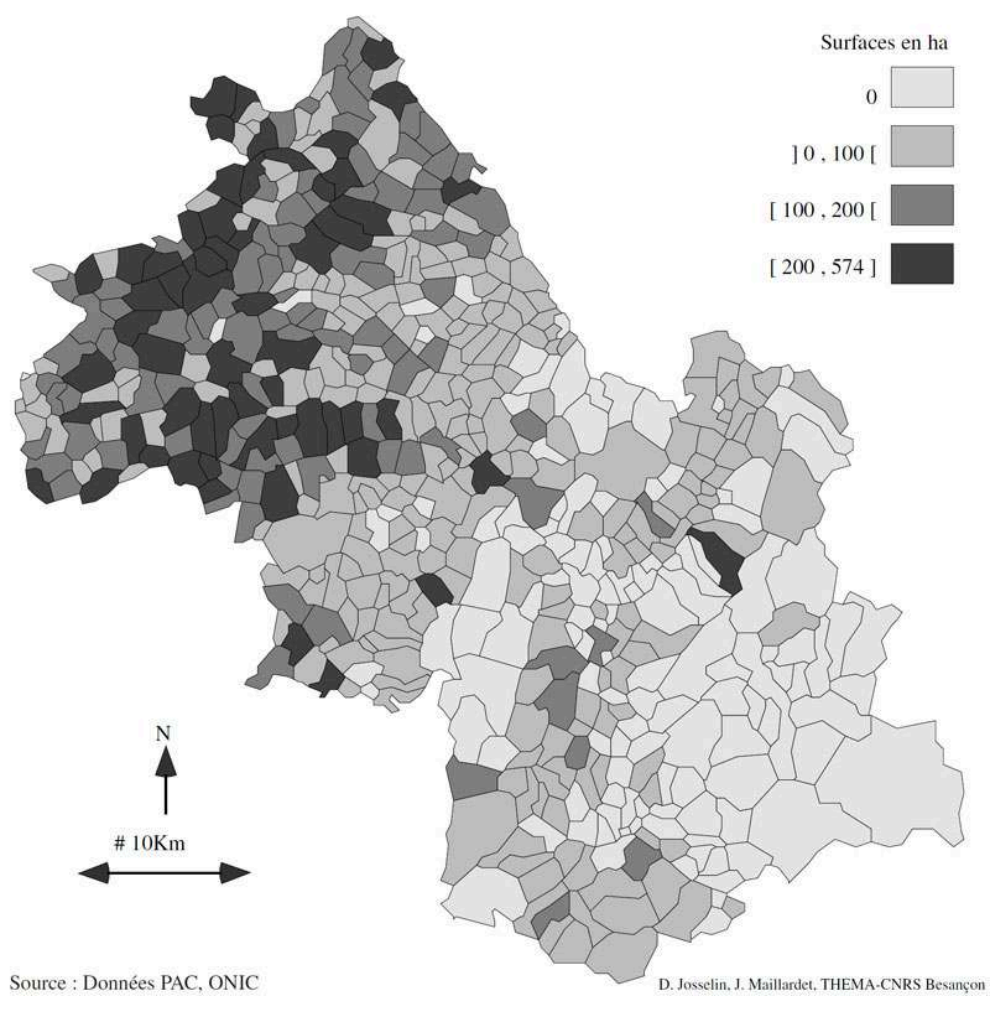

Données PAC, dossiers « jaunes ».

« $\mathrm{n}$ hectares de terres sont exploités dans une commune donnée par des agriculteurs ayant leur siège administratif dans d'autres communes ».

Fig. 5 : Répartitions spatiales des exploitations de Tullins-Vinay, Isère (en \% des effectifs)

dans les contreforts

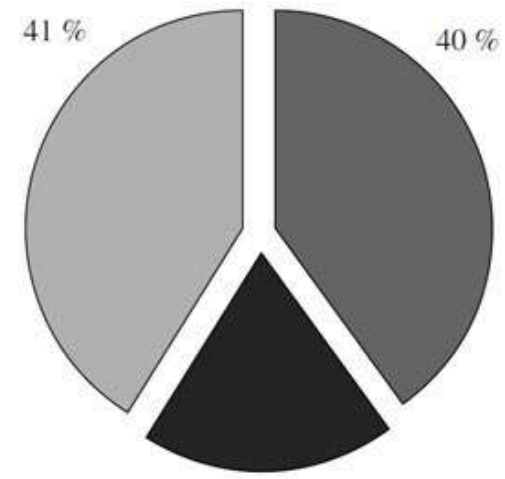

$19 \%$ dans les fonds de vallées

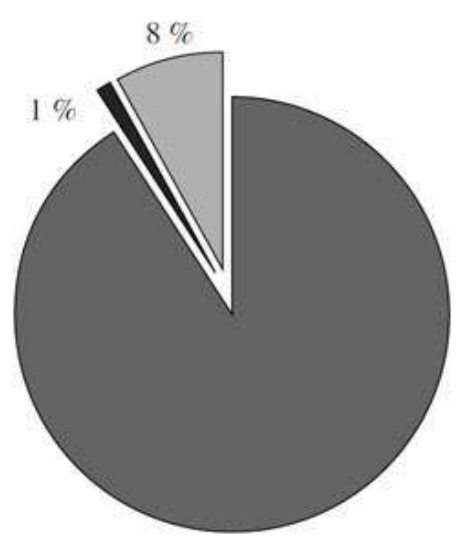

$91 \%$

exploitations en "fond de vallée"

exploitations en coteaux

exploitations transversales

Ainsi, la statistique communale peut permettre de mettre en évidence l'importance des flux agricoles et quelques uns de leurs grands traits. D'une part, les flux semblent avoir une portée limitée et sont généralement centrés sur la commune. Cette constatation 
sera à relativiser lorsque nous posséderons les informations sur les zones de montagnes (transhumance). D'autre part, il semble exister une dualité montagne-plaine, les exploitations de plaine étant plutôt consommatrices d'espaces en altitude (utilisation d'estive, pour celles qui sont orientées vers l'élevage). Enfin, les productions agricoles dominantes et les contraintes topographiques semblent avoir un rôle dans l'organisation spatiale des exploitations. Globalement, dans le département de l'Isère, on estime (en moyenne) à environ $30 \%$ la part de flux sortants par commune. Même si le découpage communal influe sur la mesure de l'emprise agricole, les flux agricoles intercommunaux constituent un premier éclairage du problème.

Pour autant, en prolongement de cette approche statistique communale, il est intéressant d'analyser la forme des flux au sein d'autres types de découpages. Nous proposons ainsi de considérer les sièges d'exploitation et les parcelles agricoles comme points de départ et d'arrivée des flux, dans un découpage dont les unités seraient des entités géographiques agricoles. Avec ce changement de grille de lecture, les flux agricoles revêtent d'autres aspects et il est alors possible de proposer des hypothèses explicatives plus proches des espaces agricoles car plus liées aux systèmes d'exploitation et à leur emprise sur le territoire.

\section{L'exploitation agricole et son parcellaire dans le territoire (Janin, 1998)}

17 Une seconde approche consiste à étudier les parcellaires des exploitations dans leur territoire. Des exemples concrets sont présentés dans une vallée de l'Isère, à l'est de la ville de Grenoble (Presage, 1994). Ils permettent de développer une analyse thématique plus approfondie des flux agricoles.

\section{A. L'exemple du Sud-Grésivaudan}

L'analyse des flux agricoles permet d'identifier des «flux transversaux » et des «flux horizontaux ».

\section{Des «flux transversaux»}

Les espaces présentant de forts contrastes entre des surfaces agricoles à potentiel très favorable (comme un fond de vallée) et des surfaces plus contraignantes (comme un coteau bordant ce fond de vallée) sont particulièrement intéressants pour analyser les flux des exploitations dans le territoire. En effet, ce fort contraste entre types de surfaces peut conduire les exploitants à avoir des stratégies très précises vis-à-vis de la valorisation de l'espace. Ainsi, lorsque des systèmes d'élevage sont présents dans ce type de contexte, ils peuvent être amenés à rechercher une complémentarité entre surfaces de potentiels différents :

- les exploitations situées pour l'essentiel en partie basse peuvent être intéressées par des pâturages sur les coteaux pour les génisses laitières ou les troupeaux allaitants, en complément de leurs surfaces intensives en zones favorables ;

- les exploitations situées plutôt sur les coteaux peuvent être intéressées par des surfaces intensifiables en fond de vallée ou plaine. 
Ces logiques génèrent des « flux transversaux » liées à la présence plus ou moins forte d'exploitations d'élevage.

Les observations faites lors d'un diagnostic territorial agricole de Tullins-Vinay (Chalier, 1996) illustrent ces remarques (fig. 5)

apparaît ainsi que, dans les contreforts composés d'une partie de fond de vallée et d'une partie de coteaux, les exploitations dites «transversales », c'est-à-dire valorisant des types de surfaces aux potentiels différents, représentent près de $40 \%$ des effectifs. A contrario, au cœur de la vallée, elles ne représentent plus que le dixième de ces effectifs.

Cela peut s'expliquer par la géomorphologie: lorsque les zones contrastées sont proches, les exploitations ont pu historiquement avoir tendance à valoriser l'ensemble de ces surfaces. Par contre, dans les territoires plus homogènes, les modes de valorisation le sont aussi.

4 Ces remarques sont confirmées par l'analyse des modes de valorisation de l'espace par les exploitations du Sud-Grésivaudan: les exploitations de grandes cultures se retrouvent essentiellement dans les fonds de vallée, tandis que celles où l'élevage domine sont plus souvent transversales ou même en coteaux (cf. fig. 6). Les exploitations mixtes " élevage et grandes cultures », ayant la plupart du temps plus de la moitié de leur SAU en grandes cultures et des systèmes fourragers comprenant du maïs ensilage ont des stratégies proches des exploitations céréalières : on les trouve essentiellement en fond de vallée ou en répartition transversale. Le cas des «systèmes noix » est particulier : ils se retrouvent dans tous les modes de répartitions spatiales dans la mesure où les noyers se plantent aussi bien en fond de vallée qu'en coteaux.

Fig. 6 : Répartition des surfaces agricoles par type de production (Sud Grésivaudan)

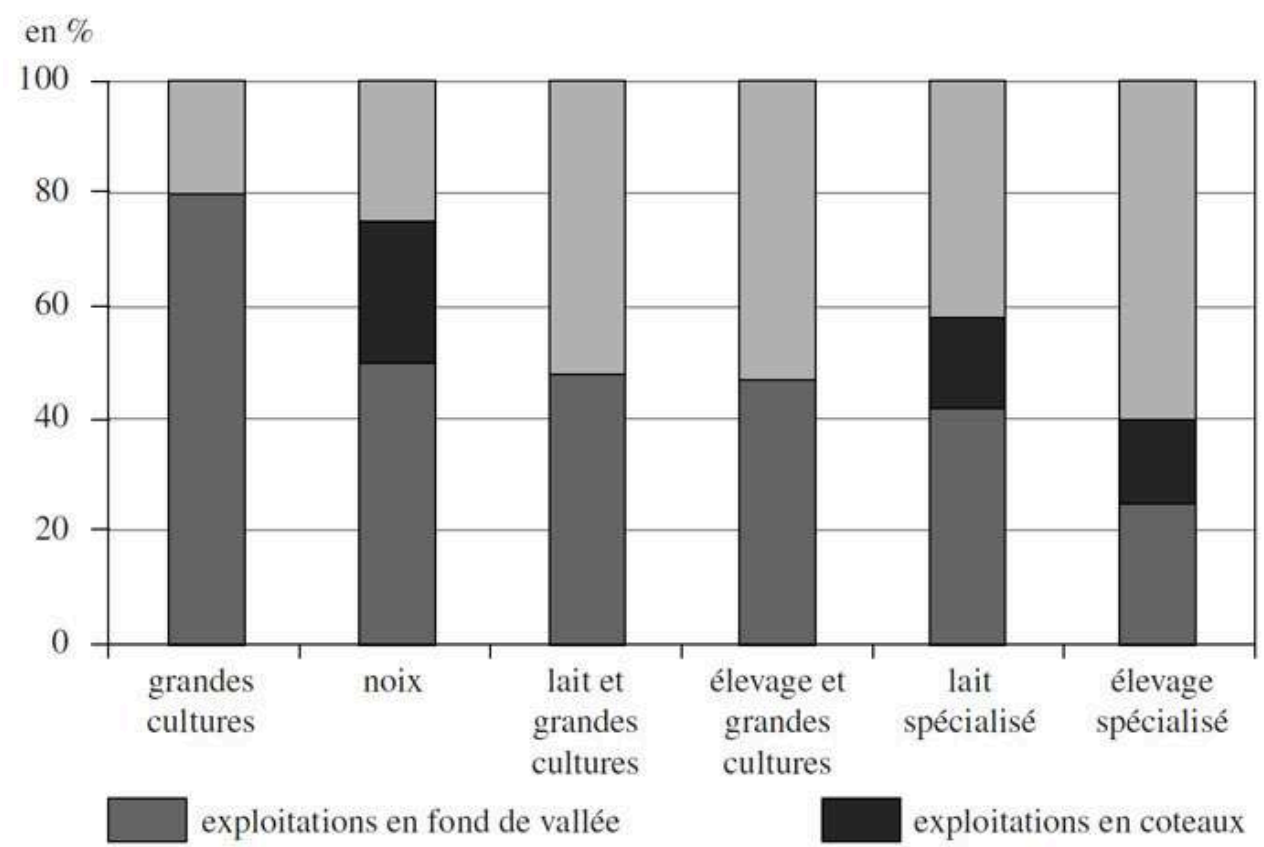

exploitations transversales 


\section{Les « flux horizontaux »}

Les exploitations de grandes cultures ont à l'évidence besoin essentiellement de terres labourables. Situées en fond de vallée, elles n'ont pas d'intérêt particulier à rechercher une complémentarité avec des surfaces situées sur les versants. Leur stratégie foncière va donc surtout consister en la recherche de terres labourables supplémentaires lorsqu'elles souhaitent augmenter leur dimension économique. Ces «flux horizontaux » sont donc générés tout d'abord par une dynamique économique locale forte, qui peut amener les exploitations à rechercher des surfaces d'autant plus loin que la pression foncière est forte dans la commune où est situé leur siège d'exploitation (fig. 7). Ainsi apparaissent des flux intercommunaux importants entre communes dans les Plaines de Bièvre et de Lyon.

Figure 7 : Flux transversaux et horizontaux dans des contreforts type « Sud-Grésivaudan »

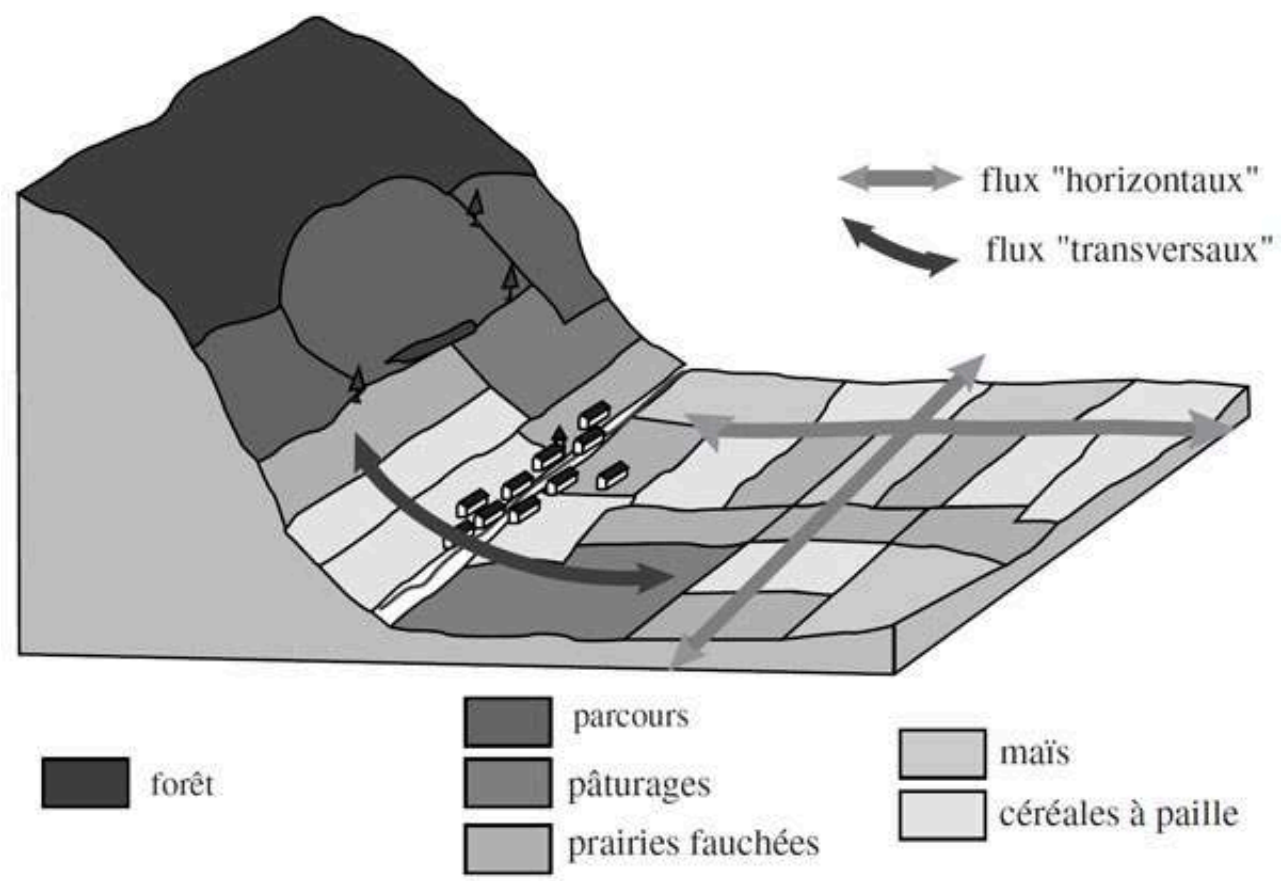

\section{B. Flux transversaux et horizontaux dans d'autres types de territoires}

Dans les territoires de montagne (fig. 8), la forte disparité entre différents types de surfaces due au relief accidenté ainsi que la présence dominante de systèmes d'élevage favorise le développement de flux « transversaux » essentiellement :

- par des stratégies de recherche de complémentarités entre surfaces pour chercher à valoriser leurs différences de potentiels: maturité plus ou moins précoce due à l'altitude ou aux expositions des versants, exploitation mécanique plus ou moins aisée des parcelles qui fait que les exploitations auront tendance à rechercher des complémentarités entre surfaces de fauche et surfaces de pâture ;

- pour des raisons dues à la géomorphologie et l'organisation spatiale, les réseaux de voirie, bien que serpentant à flanc de versants à cause du relief accidenté, descendent logiquement vers le fond de vallée. Les exploitations peuvent donc être amenées à 
rechercher leurs surfaces le long de ces voies. Il leur est ainsi souvent plus aisé d'exploiter des parcelles du versant opposé que des parcelles de leur versant mais desservies par un autre chemin.

Fig. 8 : Flux transversaux et horizontaux

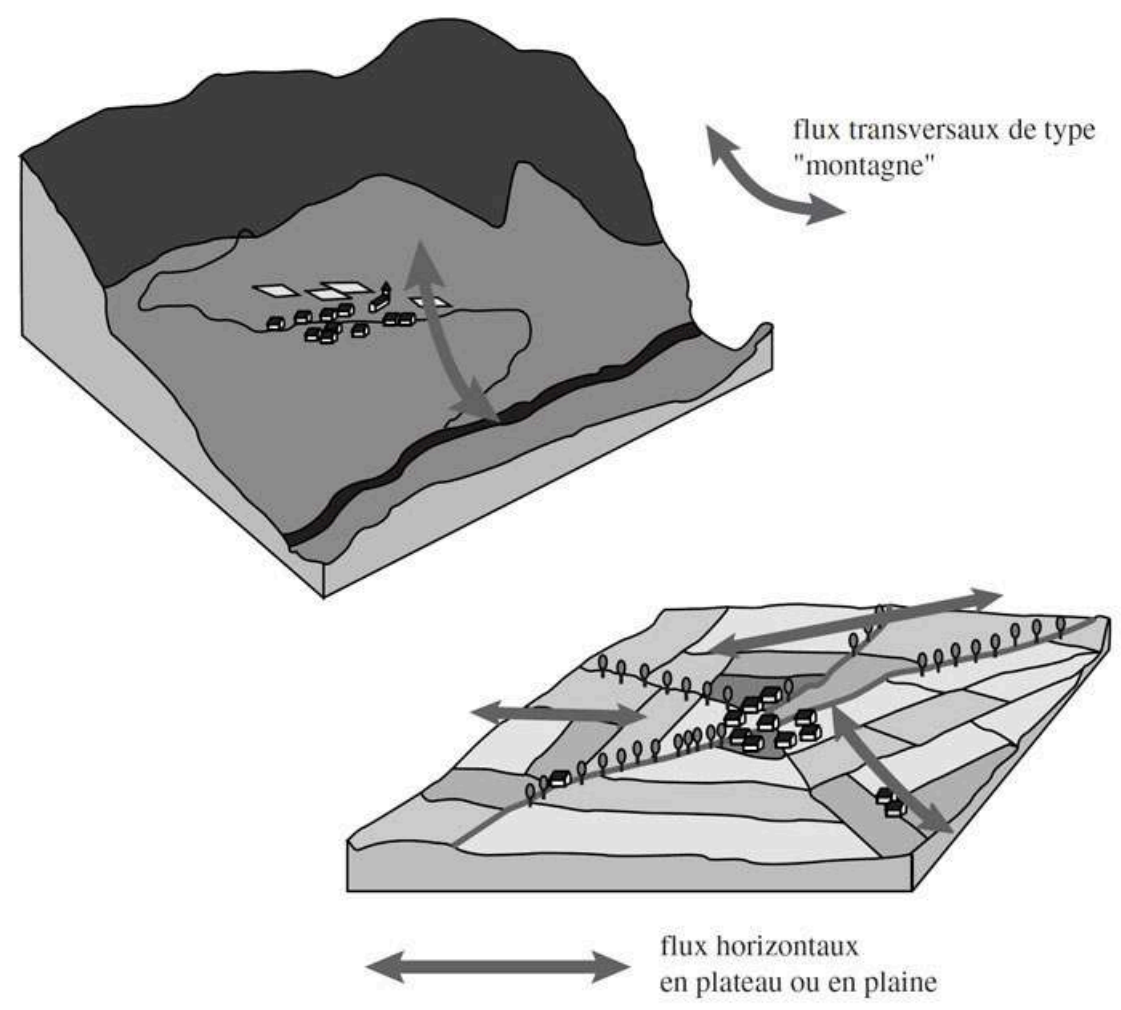

Dans les territoires de plaine ou de plateaux plus homogènes en termes de contraintes d'utilisation des sols, les flux des exploitations apparaissent guidés essentiellement par des stratégies de développement économique, les différences de qualité agronomique des sols intervenant secondairement. Les exploitations de grande culture sont plus mobiles par leurs pratiques que les fermes d'élevage.

\section{Dynamiques d'évolution des flux : éléments d'analyse}

D'après les premiers éléments d'analyse, l'évolution des flux apparaît en premier lieu liée à des dynamiques propres à l'espace agricole. Celles-ci peuvent être d'abord d'ordre fonctionnel, et concerner essentiellement les «flux transversaux». Par exemple dans le cas de territoires comme les "contreforts " illustrés par le SudGrésivaudan, les exploitations situées en zones difficiles peuvent avoir tendance à développer ces flux par l'agrandissement en fond de vallée afin d'augmenter leur proportion en surfaces favorables. A contrario, les exploitations situées originellement en fond de vallée peuvent être amenées à délaisser peu à peu les surfaces de coteaux pour intensifier les fonds de vallée lorsqu'elles ont l'opportunité de s'y agrandir. Il peut alors se produire une disparition de leur transversalité au profit d'une localisation en "fond de vallée » qui se traduit dans le paysage par un glissement des lisières forestières vers le bas des pentes. 
31 Les "flux transversaux" peuvent aussi se réduire par évolution structurelle des exploitations. Dans des territoires comme la Bièvre, de grandes exploitations mixtes en « élevage et grandes cultures » évoluent vers un abandon des ateliers d'élevage lors d'agrandissements permettant d'atteindre une viabilité économique par les cultures. Cette tendance est quelque peu freinée par les droits de produire que certaines exploitations souhaitent préserver. Malgré tout, elle peut générer la régression des «flux transversaux » et l'abandon progressif des coteaux ou versants par l'agriculture.

L'évolution des flux peut aussi être liée à des événements extérieurs à l'espace agricole (Thinon, 1996). Par exemple, le développement d'espaces urbanisés continus entre des versants et un fond de vallée, ou la création d'une infrastructure importante peut amener les agriculteurs à délaisser peu à peu les flux transversaux qui obligent à traverser ces espaces urbanisés.

\section{Conclusion : éléments d'analyse des flux agricoles}

D’un point de vue thématique, certains enseignements peuvent être tirés :

- globalement, les flux agricoles sont centrés sur la commune, autour de l'exploitation agricole ; plus on s'éloigne du siège de l'exploitation $A$ et moins on a de chance de trouver des parcelles exploitées par A ;

- les flux agricoles sont plus importants dans les territoires de systèmes de grandes cultures à partir de communes où les pressions foncières sont fortes. Les stratégies structurelles de développement économique paraissent alors dominantes ;

- ils sont denses aussi dans les territoires contrastés (type montagne ou contreforts) où les agriculteurs ont tendance à rechercher dans une logique fonctionnelle les complémentarités entre potentiels de surfaces différents ;

- les limites naturelles semblent a priori contenir l'expansion des flux. Il conviendrait d'évaluer dans quelles mesures ces contraintes ont une influence sur la structure des flux.

D'un point de vue méthodologique, il peut être intéressant de comparer les deux modes de représentation des flux agricoles que nous venons de présenter, chacun ayant ses avantages et ses inconvénients.

Le premier mode fait intervenir la partition communale. L'entité géographique en présence est la commune. On ne sait pas exactement où se trouvent les parcelles exploitées ni les sièges dans le territoire de celle-ci. En revanche, on connaît la valeur des flux. Le découpage administratif permet de disposer sans trop de difficultés des informations, car elles obéissent aux contraintes de confidentialité. Sur la base de ce découpage, il est ainsi possible de réaliser des cartes de flux et d'analyser leur structure, dans un espace contraint par une partition. Mais il est plus délicat de tenter des interprétations thématiques, notamment au sujet d'éventuelles causalités sur la répartition des flux. L'ampleur du phénomène, si l'on dispose de l'ensemble des informations utiles, peut être évaluée et la structure des flux intercommunaux peut être étudiée.

La seconde représentation change radicalement d'échelle d'appréhension et nécessite une connaissance du parcellaire de l'exploitation étudiée. Il s'agit d'une information délicate à utiliser sur de grands espaces, car impossible à recenser de manière exhaustive et tombant sous le joug des contraintes de confidentialité. Son utilisation est pertinente indépendamment des découpages administratifs. Paradoxalement, son 
analyse prend tout son intérêt à l'échelle des entités géographiques agricoles, voire des unités paysagères, permettant une analyse fine et thématique des flux.

Le point commun entre ces deux approches complémentaires réside dans la notion de flux agricole. Le flux revêt une composante statique (un flux est une surface agricole) et dynamique (un flux contient intrinsèquement des mouvements entre un point de départ et d'arrivée). Nous pensons que l'analyse de l'emprise agricole peut reposer sur ces deux composantes. La première permet de disposer d'une information globale à l'échelle de la commune. Cette information peut être dérivée d'une analyse plus fine à l'échelle du parcellaire. Mais ce dispositif ne pourra être mis en place que dans certains secteurs agricoles dans le cadre d'actions impulsées notamment par les Chambres d'Agriculture. D'où l'intérêt de chercher à dégager des formes standards des flux agricoles à l'échelle de l'exploitation et de son parcellaire afin d'en extrapoler les caractéristiques à d'autres échelles, comme celle de la commune.

\section{BIBLIOGRAPHIE}

Benoit M., Deffontaine J.-P., Moisan H. (1982). - Activités agricoles, espace, parcelles et paysages. Doc. INRAP $n^{\circ} 29$.

Benoit M. (1985) - La gestion territoriale des activités agricoles. L'exploitation agricole et le village: deux échelles d'analyse. Thèse INRA-SAD.

Caro P., Saint-Julien T. (1997). - Emplois et entreprises, Atlas de France, vol. 3, Paris, La Documentation Française, 128 pages.

Cavailhes J., Normandin D. (1992). - Les sources statistiques sur l'utilisation du territoire et son évolution, Document de travail, INRA, 77 pages + annexes.

Chalier S. (1996). - Diagnostic territorial du Pays de Tullins-Vinay. Chambre d'Agriculture de l'Isère/ ADASEA.

DRAF (1994). - Risques de déprise agricole. Localisation en région Rhône-Alpes, Lyon, DRAF RhôneAlpes, 60 pages.

Janin C. (1995). - Peut-on faire l'économie du paysage pour gérer le territoire ? in Dossier de Revue de Géographie Alpine $\mathrm{n}^{\circ} 15$ L'agriculture dans le Paysage, une autre manière de faire $\mathrm{du}$ développement local, pp. 11-31.

Janin C. (1997). - Paysage et pays : le sens du développement local ? Thèse au Laboratoire de la Montagne Alpine (CNRS URA 344, Grenoble), 220 p. + annexes.

Janin C. (1998). - Typologie Paysages et Agricultures. Guide méthodologique d'une analyse de la contribution de l'agriculture à la gestion de l'espace local. PRESAGE/ Chambre d'Agriculture de l'Isère/ Chambre Régionale Rhone-Alpes.

Josselin D. (1995). - La déprise agricole en zone de montagne. Vers un outil d'aide à la modélisation spatiale couplant Systèmes d'Induction et d'Information Géographique, Thèse de Doctorat de Géographie, Grenoble, 392 pages + annexes. 
Josselin D., Laurent C. (à paraître). - Comment mesurer l'emprise agricole sur le territoire français, actes du colloque ThéoQuant, Besançon, 1997.

Laurent C. (1994). - L'agriculture paysagiste : du discours aux réalités, in Natures-Sciences-Sociétés $n^{\circ} 2-3$, pp. 231-241.

Laurent C., Mundler P. (1995). - L'agriculture et le territoire de la commune de Pajoux, en Isère, portrait d'une situation ordinaire. Ou, de la difficulté de mesurer la dynamique de l'emprise de l'agriculture sur le territoire, INRA Versailles/CFA Anjou, 22 pages + annexes.

Louault F. (1982). - La délimitation des régions agricoles in Norois, $\mathrm{n}^{\circ}$ 115, juillet-septembre 1982, pp. 345-368.

Mergoil G., Roudie P. (1991). - Friches et recensements de l'Agriculture, in Revue de Géographie de Lyon, Vol. 66, $\mathrm{n}^{\circ}$ 1, pp. 5-10.

PRESAGE (1994). - Ils valorisent mieux l'espace. 10 cas d'exploitations agricoles en Rhone-Alpes. Institut de l'Elevage et Chambre Régionale d'Agriculture Rhone-Alpes, 46 pages.

Saillant S. (1996). - La cohérence spatiale des mesures PAC en question. Le cas du gel des terres en Isère, ENSAD, INRA-SAD, Mémoire de fin d'étude ENSIAA, 62 pages + annexes.

Schmitt B. (1992). - Un découpage de l'espace en aires de déplacement domicile-travail en vue de l'étude du développement économique des espaces ruraux, Notes de travail, INRA-ESR, Dijon.

Thinon P. (1996). - Relations territoire-agriculture et urbanisation. Recherche d'unités de gestion territoriale. Le cas du Vexin français. INRA-SAD Versailles.

\section{RÉSUMÉS}

Parallèlement à une diminution du nombre d'agriculteurs sur le territoire français, les exploitations agricoles sont de plus en plus consommatrices d'espace. Cela induit une dispersion accrue des surfaces exploitées. Ainsi, une même structure agricole peut exploiter des surfaces éloignées de son siège administratif, parfois dans d'autres communes. Il se crée ainsi des «flux agricoles ». La quantification de ces flux répartis dans l'espace géographique, dépend des objets géographiques qu'ils relient (commune, siège d'exploitation, parcelle agricole ou cadastrale) et $\mathrm{du}$ découpage territorial considéré, support de l'analyse. Ce peut être la partition communale, comme tout autre découpage thématique. Cet article propose une évaluation de l'ampleur du phénomène de flux agricoles à l'aide des données disponibles à l'échelle de la commune, une définition de ces flux ainsi que quelques hypothèses explicatives de ces flux à l'échelle du territoire des exploitations.

There is a paradox : the number of farmers on French territory is decreasing while the space consumed by farming concerns is increasing. This induces an important dispersion of agricultural lands. Thus, any farmer can use lands far from his administrative headquarters, sometimes in other communes. This defines what we call «agricultural flows». The quantification of these flows, often ditributed in geographical space, depends on the geographical objects to which they refer (commune, administrative location, agricultural or cadastral parcel) and on the territorial partition considered, support for the analysis. This paper proposes: a definition of these agricultural flows, an evaluation of the extent of the phenomenon, using available data, some theoretical explanations about their existence at commune scale. 
Gleichzeitig zur Abnahme der Zahl der Landwirte in Frankreich steigt der Landverbrauch der Agrarbetriebe mehr und mehr. Damit verbunden ist eine steigende Zerstreuung der Nutzflächen. So kann ein Betrieb weit von seinem Sitz entfernte Flächen nutzen, manchmal in anderen Gemeinden. So entstehen fliessende Veränderungen in der Landwirtschaft. Die Quantifizierung dieses «Flusses" im geographischen Raum hängt von den diesbezüglichen geographischen objekten ab (Gemeinde, Sitz des Betriebs, Nutzungs- oder Kadasterparzelle) und von dem administrativen Zuschnitt als Grundlage der Analyse. Dies kann die kommunale Gliederung wie jeder andere thematische Zuschnitt sein. Dieser Artikel beabsichtig eine Einschätzung zum Umfang dieses Phänomens eines Flusses mit Hilfe verfügbarer Daten auf Gemeindeebene wie auch eine Definition und erklärende Hypothesen zu diesen Flüssen auf Betriebsebene.

\section{INDEX}

Mots-clés : commune, découpage territorial, exploitation agricole, flux agricoles, parcellaire Keywords : agricultural flows, agricultural structure, french commune, parcels, spatial partitions

Schlüsselwörter : Agrarbetrieb, Fliessende Besitzverteilung in der Landwirtschaft, Parzellenstruktur, Territoriale Gliederung

\section{AUTEURS}

DIDIER JOSSELIN

THEMA, UPRESA 6049 - Université de Franche-Comté - 32, rue Mégevand 25030 Besançon

\section{CLAUDE JANIN}

Chambre d'Agriculture de l'Isère - LAMA-CNRS, Grenoble

\section{JÉRÔME BOLOT}

THEMA, UPRESA 6049 - Université de Franche-Comté - 32, rue Mégevand 25030 Besançon - INRASADIF, Versailles 